J Am Chem Soc. 2019 March 20; 141(11): 4711-4720. doi:10.1021/jacs.8b13613.

\title{
Biomolecular Solvation Structure Revealed by Molecular Dynamics Simulations
}

\author{
Michael E. Wall ${ }^{1,{ }^{*}}$, Gaetano Calabró ${ }^{2,3}$, Christopher I. Bayly², David L. Mobley ${ }^{3,4}$, and \\ Gregory L. Warren ${ }^{2}$ \\ ${ }^{1}$ Computer Computational, and Statistical Sciences Division, Los Alamos National Laboratory, \\ Mail Stop B256, Los Alamos, NM 87545 USA \\ 2OpenEye Scientific Software, 9 Bisbee Court, Unit D, Santa Fe, NM 87507 \\ ${ }^{3}$ Department of Pharmaceutical Sciences, University of California, Irvine, 3134B Natural Sciences \\ 1, Irvine, CA 92697 USA \\ ${ }^{4}$ Department of Chemistry, University of California, Irvine, 3134B Natural Sciences 1, Irvine, CA \\ 92697
}

\section{Abstract}

To compare ordered water positions from experiment with those from molecular dynamics (MD) simulations, a number of MD models of water structure in crystalline endoglucanase were calculated. The starting MD model was derived from a joint X-ray and neutron diffraction crystal structure, enabling the use of experimentally assigned protonation states. Simulations were performed in the crystalline state, using a periodic $2 \times 2 \times 2$ supercell with explicit solvent. Water Xray and neutron scattering density maps were computed from MD trajectories using standard macromolecular crystallography methods. In one set of simulations, harmonic restraints were applied to bias the protein structure toward the crystal structure. For these simulations, the recall of crystallographic waters using strong peaks in the MD water electron density was very good, and there also was substantial visual agreement between the boomerang-like wings of the neutron scattering density and the crystalline water hydrogen positions. An unrestrained simulation also was performed. For this simulation, the recall of crystallographic waters was much lower. For both restrained and unrestrained simulations, the strongest water density peaks were associated with crystallographic waters. The results demonstrate that it is now possible to recover crystallographic water structure using restrained MD simulations, but that it is not yet reasonable to expect unrestrained MD simulations to do the same. Further development and generalization of MD water models for force field development, macromolecular crystallography, and medicinal chemistry

\footnotetext{
*Corresponding Author Michael E. Wall, Computer Computational, and Statistical Sciences Division, Los Alamos National Laboratory, Mail Stop B256, Los Alamos, NM 87545 USA, TEL: 505-665-4209, mewall@lanl.gov.

Author Contributions

The manuscript was written through contributions of all authors. All authors have given approval to the final version of the manuscript.

Los Alamos National Laboratory LA-UR-18-31234

Supporting Information

Supporting Fig. S1 shows the dependence of precision and recall of the MD water density peaks on the peak height threshold. Crystal structure .pdb coordinates and structure factor .mtz files are provided in a .tgz archive. After the archive is expanded, Coot may be launched in the supporting_info/ directory to compare the crystal structure to MD water densities, using a saved state. File descriptions are in the README file in the directory.
} 
applications is now warranted. In particular, the combination of room-temperature crystallography, neutron diffraction, and crystalline MD simulations promises to substantially advance modeling of biomolecular solvation.

\section{Graphical Abstract}

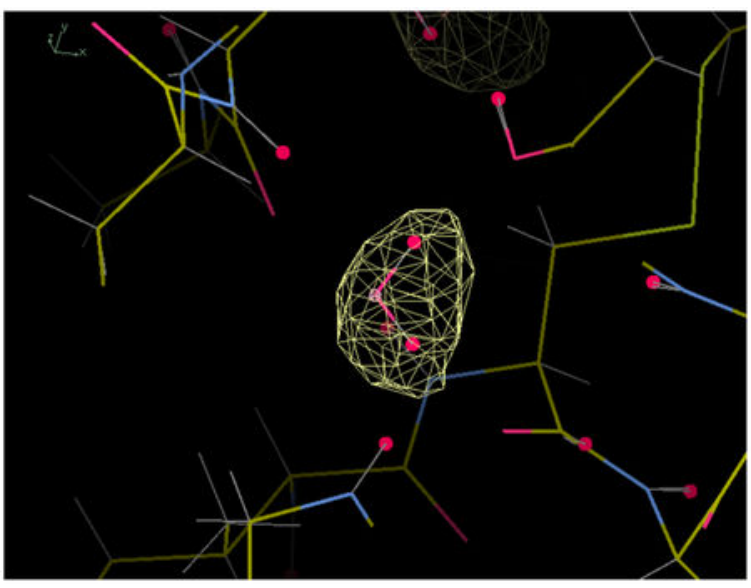

\section{Keywords}

Water structure; molecular dynamics simulation; protein crystallography; neutron diffraction

\section{INTRODUCTION}

Solvent structure is very important in biomolecular simulations for medicinal chemistry applications. In protein crystallography, ordered water molecules give rise to regions of elevated solvent density. In these regions, the interactions between a solvent water molecule and the biomolecular environment are sufficiently strong to overcome the translational and rotational entropies favoring diffusion. Computational methods have been developed for modeling water to approximate the energetic contributions of such interactions ${ }^{1-3}$. For example, ligand-binding free energies calculated from simulations are especially sensitive to the details of water structure and interactions in the immediate neighborhood of the binding site ${ }^{4-5}$. The accuracy of these calculations depends critically on the quality of the water model. Although not a direct measure of these interactions, the ability of an MD simulation to recover experimentally determined ordered waters is a useful surrogate measure of water model quality in the biomolecular environment.

An early MD simulation of a hydrated periodic unit cell of crystalline pancreatic trypsin inhibitor marked an important step in modeling water structure ${ }^{6}$; however, only $19 \%$ (9 of 47) of the crystallographic waters were within $1 \AA$ of a water density peak in the MD model. Although important studies of MD predictions of water structure followed (see review by Pettitt, Makarov, and Andrews ${ }^{7}$ ), few studies since have been carried out to validate detailed water structure predicted from MD simulations using strict comparisons to crystallography data. Higo and Nakasako ${ }^{8}$ compared a calculation of solution MD water density to a crystal structure of lysozyme; using a density threshold of 0.06 molecules/Å to reject peaks, 
approximately $60 \%$ of waters were within $1.4 \AA$ of a MD water density peak (estimated from Table 1 and Fig. 4 in Higo and Nakasako ${ }^{8}$ ). Altan et al. ${ }^{9}$ used a crystalline MD approach to model water structure in $\mathrm{Yb}^{3+}$-substituted mannose-binding protein (MBP); at the weakest density threshold of $0.6 e^{-} / \AA^{3}$ (roughly equivalent to the above density of 0.06 molecules $/ \AA^{3}$, assuming $10 e^{-}$/molecule), $70 \%$ of crystallographic waters were within $1.4 \AA$ of a MD water density peak (from Fig. 4 in Altan et al. ${ }^{9}$ ).

Here we present models of water structure for endoglucanase (EG) from Phanerochaete chrysosporium, obtained using a crystalline MD approach that was designed specifically for comparisons to X-ray diffraction data ${ }^{10}$. The choice of EG as a model system addressed several issues with the related MBP study: the MBP model was derived from X-ray diffraction data, protonation states were assigned without experimental data, and the structure was obtained from crystals at $110 \mathrm{~K}$ rather than room temperature. In contrast, the EG crystal structure (Protein Data Bank ${ }^{11}$ entry $3 \mathrm{X} 2 \mathrm{P}$ ) was obtained using both X-ray and neutron diffraction data ${ }^{12}$. The use of neutron diffraction data enables improved modeling of water structure ${ }^{13}$ and the use of experimentally assigned protonation states ${ }^{14}$ (see also reviews ${ }^{15-16}$ ). In addition, the EG crystal structure was obtained at room temperature. Temperature differences can influence the water structure in protein crystals, as observed using neutron diffraction ${ }^{13}$.

We found that MD simulations of crystalline EG with protein and ligand non-hydrogen atom restraints had very good recall of crystallographic waters, with substantial agreement between the water orientations from the neutron scattering density and the MD model. For both restrained and unrestrained simulations, the strongest water density peaks were associated with crystallographic waters. However, the unrestrained MD model had poor recall of crystallographic waters. It is therefore now possible to recover crystallographic water structure using restrained MD simulations, but it is not yet reasonable to expect unrestrained MD simulations to do the same. The amount of effort and computational resources required make it possible to apply the present approach to many other systems. Water structure appears to provide a sensitive test of MD simulations, with potential to advance force field development. Together, the combination of room-temperature crystallography, neutron diffraction, and crystalline MD simulations can improve understanding of biomolecular solvation.

\section{METHODS}

\section{Model systems.}

We prepared a modified model of the endoglucanase-cellopentaose complex from Phanerochaete chrysosporium ${ }^{12}$. To build the model, Protein Data Bank ${ }^{11}$ entry 3X2P was re-refined using the published $1.5 \AA$ neutron and 1.0 $\mathrm{A} \mathrm{X}$-ray diffraction data. Joint X-ray and neutron refinement was performed using phenix.refine ${ }^{17}$ version 1.143211 . The structure was modeled using either the native form of Asn92 or the imidic acid form that was used in the published structure ${ }^{12}$. We found insufficient crystallographic evidence to support the imidic acid form and therefore used the native form for the simulations. Protonation states for protein exchangeable H/D atoms were modeled using the neutron scattering density, considering the chemical environment. In some cases (Asp-Arg and Glu- 
Arg salt bridges; His residues), the model was modified with respect to Nakamura et al. ${ }^{12}$. $\mathrm{H} / \mathrm{D}$ atoms were added to waters as appropriate considering evidence from the neutron scattering density and H-bond interactions with the local environment.

Two alternative solvent models were used: water with elemental $\mathrm{Na}+$ and $\mathrm{Cl}$ - ions $(\mathrm{NaCl})$ and water with 2-Amino-2-(hydroxymethyl)propane-1,3-diol (Tris+) and $\mathrm{Cl}$ - ions (Tris-Cl). The Tris-Cl solvent model was developed to address the issue of ordered $\mathrm{Na}+$ potentially interfering with water structure in the $\mathrm{NaCl}$ simulation (Results). In the $\mathrm{NaCl}$ model, the system was solvated with water, $\mathrm{Na}+$ counterions were added to neutralize, additional $\mathrm{Na}+$ and $\mathrm{Cl}$ - ions were added in a 1:1 ratio to match the $50 \mathrm{mM}$ Tris-Cl concentration of the mother liquor. In the Tris- $\mathrm{Cl}$ model, the $\mathrm{Na}+$ ions were replaced by explicit Tris+ ions.

The protonation state of HIS residues was determined using the neutron diffraction data (see above). On this basis, the imidazole moiety of His64 was modeled in the uncharged deltatautomer (residue name HID), His 107 and His130 were modeled in the charged tautomer (residue name HIP), and His112 was modeled in the uncharged epsilon-tautomer (residue name HIE). Deuterium atoms were modeled into the crystal structure at exchangeable hydrogen positions using an alternative conformation syntax; these were stripped from the structure, so that only the hydrogens remained. Alternative conformations of side chains were trimmed to just the " $\mathrm{A}$ " conformation.

The crystalline models were prepared by using the $\mathrm{P} 2{ }_{1}{ }_{1} 2_{1}$ space group to expand the asymmetric unit of the protein-ligand complex to the P1 unit cell and creating a $2 \times 2 \times 2$ supercell. GROMACS ${ }^{18}$ version 5.1.4 tools were then used to prepare MD models. The protein structures were parameterized using amber99sb-ildn ${ }^{19}$, water was modeled using TIP3 $\mathrm{P}^{20}$, and the Tris+ was modeled using GAFF parameters ${ }^{21}$. The ligand was added using GLYCAM $^{22}$ parameters, with appropriate edits to the amber99sb-ildn parameter files. A right rectangular simulation box was defined with dimensions $a=92.956 \AA, b=117.270 \AA$, $c=129.488 \AA$ - double the unit cell dimensions.

At this point the building of the $\mathrm{NaCl}$ and Tris- $\mathrm{Cl}$ models diverged. For the $\mathrm{NaCl}$ model, the void volume of the crystalline system was filled with TIP3P waters ( $g m x$ solvate). To create a salt concentration reflecting the $50 \mathrm{mM}$ Tris- $\mathrm{Cl}$ in the mother liquor, the number of solvent atoms associated with the void volume was estimated as $\mathrm{N}=62,865$ using a trial solvation. Based on this number, 40 waters were replaced by Na+ ions (gmx genion). To account for the $\mathrm{Cl}$ - associated with the Tris+, 40 waters were replaced by $\mathrm{Cl}$ - ions. Then, to neutralize the system, 96 additional waters were replaced by $\mathrm{Na}+$ ions, to bring the total to 136 (gmx genion).

For the Tris-Cl model, the $136 \mathrm{Na}+$ ions were replaced by explicit Tris+ ions. First, 136 Tris + ions were added to the void volume (gmx insert-molecules). The remainder of the void was filled with TIP3P waters ( $g m x$ solvate). Then, 40 waters were replaced by Cl- ions ( $g m x$ genion).

The number of waters in each of the models was determined as the result of applying a procedure for achieving near atmospheric pressure in crystalline NVT simulations ${ }^{23}$. Iterative application of energy minimization, NVT simulation, and solvation was performed 
until a mean pressure in the range $(-100,+100)$ bar was achieved. For the $\mathrm{NaCl}$ model, the number of waters was increased by 2,200 to 23,258 . For the Tris- $\mathrm{Cl}$ model, the number of waters was increased by 2,300 to 22,568 .

The final compositions of the systems used for production simulations are as follows. The $\mathrm{NaCl}$ model had 32 copies of the protein+ligand complex; 23,258 TIP3P waters; $136 \mathrm{Na}+$ ions; and $40 \mathrm{Cl}$ - ions. A total of 150,910 atoms were distributed as follows: 77,504 protein atoms; 3,456 ligand atoms; 69,774 water atoms; and 176 ions. The Tris-Cl model had 32 copies of the protein+ligand complex; 136 Tris+ ions; 22,568 waters; and $40 \mathrm{Cl}$ - ions. A total of 151,424 atoms distributed as follows: 77,504 protein atoms; 3,456 ligand atoms; 2,720 Tris atoms; 67,704 water atoms; and 40 chloride ions. The $\mathrm{NaCl}$ model is illustrated in Fig. 1.

\section{MD simulations.}

Simulations were performed in GROMACS version 5.1.4 ( $\mathrm{NaCl}$ model) and GROMACS version 2018 (Tris- $\mathrm{Cl}$ model) using the leap-frog integration method with a $2 \mathrm{fs}$ time step. Fourth order holonomic LINCS constraints were used for all bonds. The Verlet neighbor list scheme was used with a cutoff of $10 \AA$ for both electrostatics and Van der Waals interactions. Long-range electrostatics were computed using the Particle-Mesh Ewald method with cubic interpolation and a $1.2 \AA$ grid. The modified Berendsen thermostat was used at $300 \mathrm{~K}$, using velocity rescaling with a $0.1 \mathrm{ps}$ time constant; the protein-ligand complex was treated as a separate temperature group from the rest of the atoms. Periodic boundary conditions were used.

For each of the systems, NVT simulations were performed in which the protein-ligand complex was harmonically restrained. The protein non-hydrogen atoms and all ligand atoms were restrained to their positions in the crystal structure itself (not the energy-minimized crystal structure) using $209.2 \mathrm{~kJ} / \mathrm{mol} \mathrm{nm}{ }^{2}$ spring constants, corresponding to $0.5 \mathrm{kcal} / \mathrm{mol}$ $\AA^{2}$. This moderate restraint addressed our concern that a stronger restraint of $1,000 \mathrm{~kJ} / \mathrm{mol}$ $\mathrm{nm}^{2}$ (the GROMACS default) would lead to artificial ordering at the solvent interface and a less realistic water structure ${ }^{24}$. Simulations were performed for both the $\mathrm{NaCl}$ and Tris-Cl models. The duration for restrained simulations was $100 \mathrm{~ns}$.

For the NaCl model, an unrestrained NVT simulation also was performed, without a harmonic restraint. An initial 100 ns equilibration was performed in which the protein nonhydrogen atoms and all ligand atoms were restrained to their positions in the energy minimized crystal structure using $1,000 \mathrm{~kJ} / \mathrm{mol} \mathrm{nm}^{2}$ spring constants. This restrained equilibration was then followed by an unrestrained continuation. The continuation was performed by using the $100 \mathrm{~ns}$ checkpoint as a starting condition and removing the harmonic restraints. The duration of the unrestrained simulation was 1 microsecond.

\section{Mean structure factors.}

Mean structure factors were calculated for $10 \mathrm{~ns}$ sections of the restrained and $100 \mathrm{~ns}$ sections of the unrestrained MD trajectories. Results presented here correspond to the last 10 $\mathrm{ns}$ of the restrained and both the first and last $100 \mathrm{~ns}$ of the unrestrained simulations. X-ray structure factors were calculated using methods previously described ${ }^{10}$. To calculate mean 
structure factors for a section of a trajectory, it was divided into $\mathrm{O}(100)$ chunks, which were processed in parallel using a cluster of Intel(R) Xeon(R) CPU E5-2695 v4 @ 2.10GHz nodes. Prior to performing the calculation, each snapshot of the trajectory was aligned to the crystal structure using the GROMACS .tpr structure file. To do this, the .tpr file was converted to a .pdb file using gmx editconf. The .pdb file was processed to ensure the coordinates reflected the connectivity of the molecules (gmx trjconv-pbc mol). The alignment was performed using the processed .pdb file as the reference structure ( $g m x$ trjconv -fit translation - pbc nojump). Aligned structures were written as multimodel .pdb files prior to processing. These .pdb files were processed using a custom $C C T B X^{25}$ script to calculate the mean structure factor to $1.5 \AA$ resolution. In the script, the complex structure factor, $f_{n}(h k l)$, for each sample $n$ is calculated at Miller indices $h k l$, and the mean value is calculated and output in a .mtz file. To enable comparisons to the crystal structure, the single unit cell dimensions and $\mathrm{P} 2{ }_{1} 22_{1}{ }^{1}$ space group were used in the .pdb CRYST1 record. Averages for longer sections of the trajectory were accumulated from sums of the smaller chunks. For the present study, a modified method was developed to calculate neutron structure factors, using the scattering tables available within $C C T B X$.

For the restrained simulations, the mean structure factor already was aligned with the crystal structure. For the unrestrained simulations, an alignment of the crystal structure to the simulated electron density was performed. To perform the alignment, $C C P 4^{26}$ molrep was used, with the crystal structure as the input .pdb and the mean structure factor as the input .mtz, using both the amplitudes and phases. Because waters were stripped in the output from molrep, the crystal structure was subsequently aligned to the output molrep.pdb file, using phenix.superpose_pdbs ${ }^{17}$, yielding an aligned structure including crystallographic waters.

\section{Quantitative comparison of MD water peaks to crystallographic waters.}

Peaks in the MD water electron density were used for comparisons to the crystallographic waters. Electron density maps were computed from the mean structure factors using phenix.mtz $2 m a p^{17}$, using the crystal structure as a reference frame. Peaks with heights sufficiently smaller than a multiple of the standard deviation of the electron density (sigma) were eliminated from comparisons. (The bulk water structure was visible at a contour level of about 0.5 -sigma for the restrained simulations and about 1-sigma for the unrestrained simulations.) For the restrained simulations, peaks in excess of 3-sigma were identified using CCP4 peakmax and the positions were output as a .pdb file. For the unrestrained simulations a 2-sigma threshold was used for peak finding instead: whereas a 3-sigma threshold produced fewer than 151 waters, a 2-sigma threshold yielded a number of peaks more comparable to the restrained simulations, and yielded water envelopes similar to the restrained simulation density at 3-sigma in size and shape. The residue numbers of the 151 waters in the crystal structure were assigned in rough order of confidence. We used both the top 100 waters (residue numbers 301-400) and all 151 waters for evaluations.

A recall statistic was defined as the fraction of crystallographic waters that have a nearby peak in the simulated water density. For the recall calculation, the positions of water density peaks were compared to positions of crystallographic waters in the asymmetric unit (the 
protein asymmetric unit was entirely contained within the P1 water density map). A custom $C C T B X$ python script was written to identify the peak nearest to each crystallographic water, producing a list of crystallographic waters, the matching peak, and the distance between the two. The recall was computed as the fraction of rows in the list having a distance less than a specified cutoff $(0.5 \AA, 1.0 \AA$, or $1.4 \AA)$.

A precision statistic was defined as the fraction of peaks in the simulated water density that have a nearby crystallographic water. For the precision calculation, the water density peaks were compared to positions of crystallographic waters in the P1 unit cell. Similar to the recall calculation, a custom $C C T B X$ python script was written to identify the crystallographic water nearest to each peak, producing a list of peaks, the matching crystallographic water from the P1 unit cell, and the distance between the two. As for the recall, the precision was computed as the fraction of rows in the list having a distance less than a specified cutoff $(0.5 \AA, 1.0 \AA$, or $1.4 \AA)$.

The dependence of the recall and precision on the water density peak threshold was calculated first by computing the statistics using the base value mentioned above, and then by calculating the statistics for incrementally higher thresholds, up to the maximum peak height value.

\section{RESULTS}

\section{Crystalline MD simulations.}

MD simulations of crystalline endoglucanase from Phanerochaete chrysosporium were performed to determine models of water structure (Methods). The starting MD model (Fig. 1) was derived from a re-refined crystal structure (Protein Data Bank ${ }^{11}$ entry $3 \mathrm{X} 2 \mathrm{P}$ ). Two alternative solvent models were used: water with elemental $\mathrm{Na}+$ and $\mathrm{Cl}$ - ions $(\mathrm{NaCl})$ and water with 2-Amino-2-(hydroxymethyl)propane-1,3-diol (Tris+) and $\mathrm{Cl}$ - ions (Tris-Cl). The Tris-Cl solvent model was developed to address the issue of ordered $\mathrm{Na}+$ potentially interfering with water structure in the restrained $\mathrm{NaCl}$ simulation (see below). For each of the systems, NVT simulations were performed in which the protein-ligand complex was harmonically restrained. The duration for restrained simulations was $100 \mathrm{~ns}$. For the $\mathrm{NaCl}$ model, an unrestrained NVT simulation also was performed, without a harmonic restraint. The duration of the unrestrained simulation was 1 microsecond.

\section{X-ray scattering density and water positions.}

Simulated electron density maps were computed from the MD trajectories (Methods). Water positions were identified using strong peaks in the simulated water density. For the restrained simulations, peaks were identified using a threshold of 3-sigma. The number of peaks found in the restrained MD water density computed from the last $10 \mathrm{~ns}$ section was: 963 peaks for the $\mathrm{NaCl}$ model, and 952 peaks for the Tris- $\mathrm{Cl}$ model. For the unrestrained $\mathrm{NaCl}$ model, peaks were identified using a threshold of 2-sigma (Methods). There were 504 peaks in the density computed from the first $100 \mathrm{~ns}$ section, and 331 peaks in the density computed from the last $100 \mathrm{~ns}$ section of the simulation. The density varied more sharply for the restrained models than for the unrestrained model. For the restrained $\mathrm{NaCl}$ model, sigma 
$=0.25 e_{\_} / \AA^{3}$ and mean $=0.56 e \_/ \AA^{3} ;$ for the restrained Tris-Cl model, sigma $=0.24 e_{-} / \AA^{3}$ and mean $=0.48 e_{-} / \AA^{3}$; for the unrestrained $\mathrm{NaCl}$ model, sigma $=0.15 e_{\_} / \AA^{3}$ and mean $=$ $0.17 e_{-} / \AA^{3}$. A decreased sigma of the unrestrained model compared to the restrained models is consistent with the increased atomic motions of the protein and ligand, which are expected to disturb the solvent structure.

Restrained $\mathrm{NaCl}$ model.-Most of the top 100 water oxygens were recovered by the restrained $\mathrm{NaCl}$ model simulation. For example, buried crystallographic water 303 is contained within a 3-sigma envelope of the MD water density (Fig. 2A,B). Extended networks of solvation also were reproduced, as in the neighborhood of water 305 (Fig. 2C,D). Fig. 3 (solid line) shows the cumulative distribution of distances from the top 100 crystallographic waters to the nearest MD water peak. A summary of the statistics is in Table I. Of the top 100 waters, $80 \%$ had a MD peak within $0.5 \AA$, $94 \%$ had a MD peak within $1 \AA$, and $98 \%$ had a MD peak within $1.4 \AA$. The waters with peaks farther than $1 \AA$ away were:

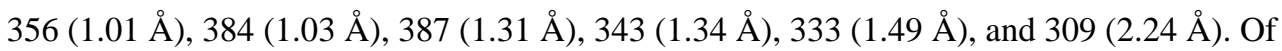
these, water 309 is the most interesting case: it was replaced by a tightly bound $\mathrm{Na}+$ ion (Fig. 4A). Water 356 is nearby and is also disturbed by the Na+ density at 309. Waters 333 and 384 are near a different peak in the $\mathrm{Na}+$ density, which appears to have disturbed the water structure in the MD. Water 343 is in a region where there is an extensive water network in the MD that is largely lacking in the crystal structure. Water 387 is a discrepancy that is not obviously related to other differences between the simulation and the crystal structure.

Restrained Tris- $\mathrm{Cl}$ model.-Because much of the disrupted water structure in the restrained $\mathrm{NaCl}$ model appeared to be associated with $\mathrm{Na}+$ density, simulations also were performed on a model in which the $\mathrm{Na}+$ ions were replaced by Tris+ ions (Model systems). The overall recovery of crystallographic waters for the restrained Tris- $\mathrm{Cl}$ model was similar to the $\mathrm{NaCl}$ model (Fig. 3, dotted line; Table I): $77 \%$ of the top 100 crystallographic waters had a MD peak within $0.5 \AA$, $95 \%$ had a peak within $1 \AA$, and $98 \%$ had a peak within $1.4 \AA$. The waters with peaks farther than $1 \AA$ away were: 343 (1.20 $)$ ), 385 (1.26 ̊) , 387 (1.30 $\AA), 398(1.41 \AA)$, and $333(1.51 \AA)$. An examination of these cases indicates that substituting Tris+ for Na+ did address some of the water structure issues, but did not lead to an improved overall recall of crystallographic waters. Importantly, water 309, which corresponds to Na+ density in the $\mathrm{NaCl}$ model, does have overlapping water density for the Tris- $\mathrm{Cl}$ model (Fig. 4B). The nearby water 359 also has overlapping density. However, water 333 is still missing in the MD, and water 384 is only marginally within the $1 \AA$ distance threshold ( $0.93 \AA$ ). Like in the $\mathrm{NaCl}$ model, the water network is extensively different in the neighborhood of 343. Water 385 is not visible at 1-sigma in the experimental $2 \mathrm{Fo}-\mathrm{Fc}$ map and is in a region where the MD otherwise agrees well with the crystal structure; it is possible that this water was incorrectly modeled using the diffraction data alone. Water 398 also is not visible, but is in a region where the MD water structure does not correspond to ordered crystallographic waters. Like for the $\mathrm{NaCl}$ model, water 387 is a discrepancy that is not obviously related to other differences between the simulation and the crystal structure. 
Unrestrained $\mathrm{NaCl}$ model.-Compared to the restrained simulation, far fewer of the top 100 crystal structure water oxygens were near MD water peaks in the unrestrained $\mathrm{NaCl}$ model. The agreement was highest in the first $100 \mathrm{~ns}$ section following the relaxation of restraints (Fig. 3, dashed line; Table I), where 25\% of crystalline waters had a MD peak within $0.5 \AA$, $50 \%$ had a MD peak within $1 \AA$, and $62 \%$ had a MD peak within $1.4 \AA$. The agreement is poorer in the last $100 \mathrm{~ns}$ section (Table I only), where $18 \%$ had a MD peak within $0.5 \AA, 42 \%$ had a MD peak within $1 \AA$, and $51 \%$ had a MD peak within $1.4 \AA$. This suggests that slow protein motions away from the crystal structure result in decreasing agreement with experimental water structure.

In cases where the crystalline water was not recovered by the unrestrained MD, the protein structure was not grossly inconsistent with the simulated protein electron density. A typical case is illustrated in Fig. 5A. Solvent-accessible water 335 has no nearby water MD density at 2-sigma, and two connected waters also are not recovered. The surrounding crystalline protein non-hydrogen atoms are mostly within the 1-sigma envelope of the protein MD density. Fig. 5B shows the less typical case of buried water 302, which has no nearby water MD density at 2-sigma. In this case not only is the protein MD density similar to the crystal structure, but also the water is hydrogen bonded to three sites on the surrounding protein. The lack of obvious clashes between the protein electron density and the crystalline water positions suggests that water structure is sensitive to relatively small changes in the protein environment (See Discussion).

Recall of all 151 crystallographic waters.-Recall statistics were lower for all 151 crystallographic waters than for the top 100 crystallographic waters (Table I, values in parentheses). For the restrained $\mathrm{NaCl}$ model (last $10 \mathrm{~ns}$ section), $66 \%$ had a MD peak within $0.5 \AA, 86 \%$ had a MD peak within $1 \AA$, and $93 \%$ had a MD peak within $1.4 \AA$. For the restrained Tris-Cl model (last 10 ns section), $64 \%$ had a MD peak within $0.5 \AA$, $85 \%$ had a MD peak within $1 \AA$, and $93 \%$ had a MD peak within $1.4 \AA$. For the unrestrained $\mathrm{NaCl}$ model (first $100 \mathrm{~ns}$ section), $21 \%$ had a MD peak within $0.5 \AA$, $46 \%$ had a MD peak within $1 \AA$, and $56 \%$ had a MD peak within $1.4 \AA$. It is possible that the MD model was not as accurate in the neighborhood of the last 51 waters, but it is also possible that there are more errors among the last 51 crystallographic waters than among the first 100 (See Discussion).

Precision of MD water density peaks.-Precision statistics were used to evaluate the confidence in using the MD water density peaks to predict locations of crystallographic waters (Methods). For the restrained $\mathrm{NaCl}$ model (last $10 \mathrm{~ns}$ section), 32\% of the MD water density peaks had a crystallographic water within $0.5 \AA, 43 \%$ had a crystallographic water within $1 \AA$, and $47 \%$ had a crystallographic water within $1.4 \AA$. For the restrained Tris-Cl model (last $10 \mathrm{~ns}$ section), $31 \%$ of the MD water density peaks had a crystallographic water within $0.5 \AA, 43 \%$ had a crystallographic water within $1 \AA$, and $48 \%$ had a crystallographic water within $1.4 \AA$. For the unrestrained $\mathrm{NaCl}$ model (first $100 \mathrm{~ns}$ section), $16 \%$ of the MD peaks had a crystallographic water within $0.5 \AA, 37 \%$ had a crystallographic water within 1 A, and $46 \%$ had a crystallographic water within $1.4 \AA$.

Tradeoff between precision and recall at higher MD water densities.-To assess whether stronger MD water density peaks were more likely to correspond to crystallographic 
waters, the precision was calculated for sets of peaks filtered using higher thresholds. The recall also was calculated using the higher thresholds, to determine the tradeoff in recovering crystallographic waters. The thresholds were varied in 100 uniform steps between the minimum (3-sigma for restrained and 2-sigma for unrestrained simulations) and maximum peak heights within each computed density map. For each of the simulations, the recall decreased with increasing threshold (Supporting Fig. S1A). While the recall decreased, the precision increased with increasing threshold, to a maximum of 100\% (Supporting Fig. S1B). Fig. 6 shows the tradeoff between precision and recall. For a given value of recall, the precision of the $\mathrm{NaCl}$ and Tris-Cl models was mostly higher than the precision of the unrestrained model (the precision of the unrestrained model slightly exceeds that of the $\mathrm{NaCl}$ model in a band at low recall). The precision of the Tris- $\mathrm{Cl}$ model was similar to that of the $\mathrm{NaCl}$ model at lower thresholds (higher recall), and was higher than that of the $\mathrm{NaCl}$ model at higher thresholds (lower recall).

\section{Neutron scattering density and water orientations.}

To assess the accuracy of the water orientations in the simulations, water neutron scattering densities for the restrained $\mathrm{NaCl}$ model were computed (Methods) and were compared to the experimental $2 \mathrm{Fo}-\mathrm{Fc}$ neutron scattering density. To calculate the simulated neutron scattering density, coordinates for water $\mathrm{H}$ and $\mathrm{O}$ atoms were extracted. Water $\mathrm{H}$ and $\mathrm{O}$ densities were individually computed using neutron scattering tables. The deuterium (D) density corresponding to the $\mathrm{H}$ atoms was calculated by re-normalizing the $\mathrm{H}$ density using the ratio of $\mathrm{D}$ and $\mathrm{H}$ scattering lengths. Finally, the water neutron scattering densities were computed as 0.8 (D density) +0.2 ( $\mathrm{H}$ density $)+(\mathrm{O}$ density $)$, to simulate the $80 \%$ deuterium conditions of the neutron crystallography experiment.

The boomerang-like water neutron scattering density from the simulations generally aligns with the experimental $2 \mathrm{Fo}-\mathrm{Fc}$ neutron scattering density; however, the simulation shows stronger ordering of H/D atoms than the experimental data, giving rise to a more extended appearance of the density. There also are examples where the orientation from the simulation differs from the experiment. An illustration of both cases is shown in Fig. 7. The orientation of water 303 (Fig. 7A,B) is reproduced by the simulation, but the H/D neutron scattering density is more ordered than in the experiment, as seen in the more extended density. The orientation of water 328 in the simulation, however, differs from the experimental model (Fig. 7C,D).

\section{DISCUSSION}

Both the recall and precision statistics for the present MD models were substantially higher than had been seen in similar MD water structure studies (compare Fig. 6 and Supporting Fig. S1 to Fig. 4 in Altan et al..$^{9}$ and Fig. 2 in Higo and Nakasako ${ }^{8}$ ). Altan et al. ${ }^{9}$ found that up to $70 \%$ of 254 crystallographic waters were within $1.4 \AA$ of a MD water peak in simulation of a unit cell of $\mathrm{Yb}^{3+}$-substituted mannose-binding protein. Using a similar peak height threshold, Higo and Nakasako ${ }^{8}$ found that $60 \%$ of 405 crystallographic waters were within $1.4 \AA$ of a MD water peak in a solution state simulation of lysozyme. For the present EG simulation, using the top 100 waters and a distance of $1.4 \AA$, the recall of the restrained 
models is $98 \%$ (Fig. 3). Using all 151 ordered waters in the crystal structure and a distance of $1.4 \AA$ instead, the recall of the restrained $\mathrm{NaCl}$ model is $93 \%$. In addition, the maximum precision of $100 \%$ found here is greater than the maximum reported by Altan et al. ${ }^{9}$ (70\%) and Higo and Nakasako ${ }^{8}(23 \%)$.

Only $50 \%$ of the top 100 waters and $46 \%$ of all 151 crystallographic waters were recovered within $1 \AA$ in the first $100 \mathrm{~ns}$ of our simulation in $\mathrm{NaCl}$ solvent done without restraints on the protein. Although this recall is substantially larger than the value of $19 \%$ at $1 \AA$ obtained for pancreatic trypsin inhibitor in van Gunsteren et al. ${ }^{6}$, it is much smaller than for the restrained $\mathrm{NaCl}$ or Tris- $\mathrm{Cl}$ model. Moreover, the recall is lower in the last $100 \mathrm{~ns}$ of the simulation. This result may indicate that current force fields are lacking or do not represent the protein in the crystal environment well, especially because we found that agreement got worse as the simulation progressed. Regardless of the cause of the inaccuracy, it is not yet reasonable to simulate an unrestrained protein, either in solution or crystalline state, and to assume that all the waters in the X-ray crystal structures would appear as ordered water density in the simulations.

Visual inspection of the environment of crystallographic waters that failed to be recovered by the unrestrained simulation revealed clues about why they might be missing.

Interestingly, there were no cases where the protein density from the simulation overlapped a crystallographic water. This indicates that the protein did not directly displace the missing crystallographic waters. However, in the neighborhood of the missing waters, there were small deviations between the MD protein density and the crystal structure, disrupting entire local networks of water molecules. These observations indicate that crystalline waters were not directly displaced by the protein in the unrestrained simulation; rather, smaller displacements of the protein caused changes in water structure. A more puzzling case is buried interior water 302, which exhibits three strong hydrogen bonds to Asp52O, Cys54O, and Cys172H, as shown in Fig.5B. Despite these strong H-bonds, the MD simulation has no corresponding MD density at 2-sigma for this water. Two of the missing water H-bonds are back-filled by interactions between Cys54O and Gly161H and between Asp52O and Arg56HE; however, the fact that this water leaves does beg the question of where it went and how it got out.

The present finding that unrestrained simulations do not reproduce a high fraction of the crystallographic water structure is interesting in light of the work of Lexa and Carlson ${ }^{24}$, who examined the influence of protein flexibility on the MixMD method for mapping ligand binding hot spots in hen egg-white lysozyme. In their study, simulations using limited protein flexibility identified too many hot spots, whereas a simulation with full flexibility identified a well-defined hot spot that overlapped the experimental multiple-solvent crystal structure results ${ }^{27}$. They found that water density computed from an unrestrained solution state MD simulation agreed with the locations of 11 high confidence crystallographic waters not involved in crystal packing interactions. Comparing the top 11 crystallographic waters in EG with the first $100 \mathrm{~ns}$ of the present unrestrained simulation, all but buried water 302 (illustrated in Fig. 5) are within $1.4 \AA$ of a MD water peak. In addition, the maximal precision values for the unrestrained and restrained MD models of EG water structure were 
similar (Fig. 6). The present results therefore appear to be consistent with the similar subset of results of Lexa and Carlson ${ }^{24}$, which primarily was concerned with predicting hot spots.

Previous studies have used a distance of $1.4 \AA$ to calculate the recall of crystallographic waters, corresponding to the radius of water ${ }^{8-9}$. For the restrained EG simulations, however, waters at a distance of $1.4 \AA$ from the peak lie outside of the 3 -sigma envelope (not shown). In contrast, a distance of $1 \AA$, as used by van Gunsteren et al. ${ }^{6}$, places the water within the 3sigma envelope of the MD water density, which is what a crystallographer might perceive as a correctly placed water. This observation suggests that using a more stringent distance threshold of $1 \AA$ is preferred over a threshold of $1.4 \AA$ when assessing the ability of the MD model to add information for crystallographic model building. The recovery of waters in this study was very good using the more stringent threshold of $1 \AA$ : the restrained MD simulations recovered $94 \%$ ( $\mathrm{NaCl}$ model) or $95 \%$ (Tris- $\mathrm{Cl}$ model) of the top 100 waters.

Because potential energies typically are very sensitive to small changes in atom positions, the criterion for accuracy of the water structure model might need to be even stricter for medicinal chemistry applications. In this case, the recall calculated using a distance of $0.5 \AA$ between a water peak and the corresponding crystallographic water (or even closer) might be more appropriate. The placement of water hydrogens also can be important for ligand binding free energy calculations. Our visual comparison of the calculated and experimental neutron scattering density shows a general alignment of water orientations. Together with the high recall of water positions, this alignment suggests that water placement and orientation in networks tend to go hand in hand.

The higher recall of crystallographic waters observed here for EG compared to previous MD water studies of lysozyme ${ }^{8}$ and $\mathrm{MBP}^{9}$ might reflect an increased accuracy of the present crystalline protein MD simulations. There are major differences between the model preparation and simulation methods used here for EG and the previous MD water studies of lysozyme ${ }^{8}$ and $\mathrm{MBP}^{9}$. The lysozyme study ${ }^{8}$ used a solution state MD model and bears little resemblance to our study. The MBP study ${ }^{9}$ used a substantially different crystalline MD model. One major difference is that the MBP model used a single unit cell, and the EG model used a $2 \times 2 \times 2$ supercell. Another is that the MBP study created a large cavity of bulk water in the unit cell through the removal of one copy of the protein, and averaged the results of four such simulations, choosing a different copy for each. In contrast, the EG simulations used all copies of the protein in the entire $2 \times 2 \times 2$ supercell, with a number of solvent molecules tuned to achieve near atmospheric pressure. (The EG model used an NVT ensemble to ensure that the dimensions of the supercell did not vary.) The same choices made for the MD model of EG were also made for a model of staphylococcal nuclease that improved the agreement with diffuse X-ray scattering data ${ }^{10}$, providing further indirect evidence for the advantages of the present MD model preparation and simulation methods.

Other factors might also be important to explain the high recall found for the present EG study. The MBP crystal structure was derived from X-ray data at a temperature of $110 \mathrm{~K}$, and the model of EG was derived from both X-ray and neutron data at $298 \mathrm{~K}$. The MBP model used GROMACS assigned protonation states (with exploration of some alternatives), and the EG protonation states were assigned based on the neutron data. Backbone carbon 
and nitrogen atoms were fixed in the MBP simulations, with $1,000 \mathrm{~kJ} / \mathrm{mol} \mathrm{nm}{ }^{2}$ restraints on the remaining non-hydrogen atoms. In the EG simulation, $209.2 \mathrm{~kJ} / \mathrm{mol} \mathrm{nm}^{2}$ restraints were used on all non-hydrogen atoms, with no additional restraints.

The number of 3-sigma peaks found in the restrained MD water density was far greater than the number of crystallographic waters (151): 963 for the restrained $\mathrm{NaCl}$ model, and 952 for the restrained Tris- $\mathrm{Cl}$ model. Many of the extra peaks in the unit cell correspond to waters in symmetry-related copies of the asymmetric unit. However, even accounting for symmetry, the precision of the MD model using the 3-sigma threshold was much less than the recall (43\% precision compared to $86 \%$ recall for the $\mathrm{NaCl}$ model using a $1.0 \AA$ cutoff). The excessive number of peaks indicates that the MD water structure is over-ordered, which is possibly a consequence of the harmonic restraints applied to the protein and ligand. A weaker restraint than used here $\left(209.2 \mathrm{~kJ} / \mathrm{mol} \mathrm{nm}^{2}\right.$ or $\left.0.5 \mathrm{kCal} / \mathrm{mol}^{2}\right)$ might yield a higher precision in prediction of crystallographic waters, perhaps at the cost of recall. The number of 2-sigma peaks in the $\mathrm{NaCl}$ model without restraints was lower than for the restrained MD, but still higher than the number of crystallographic waters: 504 peaks in the first $100 \mathrm{~ns}$ section, and 331 in the last $100 \mathrm{~ns}$ section. This means that water is less ordered in the unrestrained than in the restrained simulations, and that the ordering is less in the last $100 \mathrm{~ns}$ section than in the first $100 \mathrm{~ns}$ of the unrestrained simulation. The recall of crystalline waters in the unrestrained simulation also is substantially poorer than in the restrained simulations. The improved recall of the restrained simulations supports the previous finding that crystal structure restraints can improve predictions for crystallographic temperature factors, solid-state nuclear magnetic resonance (NMR) chemical shifts, and NMR backbone order parameters ${ }^{28}$. These results indicate that the MD model, possibly including the potentials, must be improved to yield accurate predictions in the absence of any restraint.

Analysis of discrepancies revealed cases where the evidence for crystallographic waters was not clear cut from the experimental data. Indeed, overinterpretation of water structure is a common pitfall in macromolecular crystallography ${ }^{29}$, and in some cases it might be unclear whether the discrepancies in this work are due to inaccuracies in the simulation or the crystal structure. The present results on EG indicate that MD models might help with this problem by providing independent evidence either for or against marginal crystallographic waters. In addition, the MD model can suggest new possible locations of waters that can then be accepted or rejected using the agreement with the diffraction data.

Filtering MD water density peaks using an increasingly high threshold decreased the recall and increased the precision of predicting crystallographic waters (Fig. 6 and Supporting Fig. S1). Until the time when the simulations and the crystallography can achieve perfect agreement, it will be necessary to consider the tradeoff between precision and recall for individual applications. For example, if the simulations are used to seek supporting evidence for crystallographic waters that have low experimental density, a high recall might be desired, calling for a low threshold. On the other hand, if the simulations are used to identify locations of tightly bound waters with high confidence, a high precision might be desired, calling for a high threshold. 
Obtaining water structure information from MD simulations requires more effort and computational resources than the usual crystallography workflow. The extra cost is not excessive, however, and the approach could be applied to many other systems. The main human effort is in setting up a complete model of the crystalline protein; the effort is increased when there are missing residues that need to be modeled in the crystal structure. The MD simulation for the present systems of $\sim 150,000$ atoms proceeded at a rate of $\sim 206$ ns/day on 16 nodes of an Intel Broadwell (Xeon E5-2695 v4 @ 2.10 GHz) cluster with 36 cores per node, yielding a $100 \mathrm{~ns}$ trajectory within a $1 / 2$ day. The same system runs at 52 ns/day on a single similar node with a NVIDIA Tesla P100 GPU, requiring two days to complete a $100 \mathrm{~ns}$ simulation. Routine MD simulations of water structure therefore are within reach, and might soon become a common step in crystallography workflows.

Previous studies have demonstrated the utility of using diffraction data to validate crystalline protein MD simulations ${ }^{10,23,30-35}$. The present study indicates that water structure can be quite sensitive to relatively small changes in the protein structure. Crystalline protein water structure studies therefore have the potential to be an especially valuable tool in validating MD simulations, e.g., for force field development.

The present results suggest that the combination of room-temperature crystallography, neutron diffraction, and crystalline MD simulations has potential to increase the accuracy of biomolecular solvation models for force field development, crystallography, and medicinal chemistry. The potential value of this combination is supported by prior experimental studies. Comparative neutron diffraction experiments on concanavalin A at room temperature and $15 \mathrm{~K}$ revealed substantial changes in water structure upon cryocooling ${ }^{13}$. In addition, crystal cryocooling was observed to change side chain conformational distributions in dihydrofolate reductase ${ }^{36}$. These studies highlight the importance of room temperature experiments for water structure studies. Room-temperature X-ray free-electron laser studies of the influenza M2 proton channel showed the sensitivity of the water structure to $\mathrm{pH}^{37}$, indicating the potential for protonation states to influence water structure. This study highlights the advantage of using neutron diffraction experiments for determining protonation states, as prediction of pKa values is notoriously difficult ${ }^{38}$. Neutron diffraction data also allow for the experimental validation of water orientations predicted by MD models, using experimentally assigned positions of the water H/D atoms.

\section{Supplementary Material}

Refer to Web version on PubMed Central for supplementary material.

\section{ACKNOWLEDGMENT}

\begin{abstract}
MW was supported by the New Mexico Small Business Assistance Program, the University of California Laboratory Fees Research Program, and the Exascale Computing Project (17-SC-20-SC), a collaborative effort of the US Department of Energy Office of Science and the National Nuclear Security Administration. GC and DM were supported by the National Institutes of Health (1R01GM108889-01). The simulations were performed using Institutional Computing machines at Los Alamos National Laboratory, supported by the US Department of Energy under Contract 89233218CNA000001.

Funding Sources
\end{abstract}


The New Mexico Small Business Assistance Program (MW), the University of California Laboratory Fees Research Program (MW), US Department of Energy (17-SC-20-SC, MW), NIH (1R01GM108889-01, GC and DM). Institutional Computing machines at Los Alamos National Laboratory are supported by the US Department of Energy under Contract 89233218CNA000001.

\title{
ABBREVIATIONS
}

\author{
MD molecular dynamics \\ MBP $\quad \mathrm{Yb}^{3+}{ }_{-}$substituted mannosebinding protein \\ EG endoglucanase \\ Tris 2-Amino-2-(hydroxymethyl)propane- 1,3-diol
}

\section{REFERENCES}

1. Abel R; Young T; Farid R; Berne BJ; Friesner RA, Role of the active-site solvent in the thermodynamics of factor Xa ligand binding. J Am Chem Soc 2008, 130 (9), 2817-31. [PubMed: 18266362]

2. Nguyen CN; Cruz A; Gilson MK; Kurtzman T, Thermodynamics of Water in an Enzyme Active Site: Grid-Based Hydration Analysis of Coagulation Factor Xa. Journal of Chemical Theory and Computation 2014, 10 (7), 2769-2780. [PubMed: 25018673]

3. Velez-Vega C; McKay DJJ; Kurtzman T; Aravamuthan V; Pearlstein RA; Duca JS, Estimation of Solvation Entropy and Enthalpy via Analysis of Water Oxygen-Hydrogen Correlations. Journal of Chemical Theory and Computation 2015, 11 (11), 5090-5102. [PubMed: 26574307]

4. Baron R; Setny P; McCammon JA, Water in cavity-ligand recognition. J Am Chem Soc 2010, 132 (34), 12091-7. [PubMed: 20695475]

5. Setny P; Baron R; McCammon JA, How Can Hydrophobic Association Be Enthalpy Driven? J Chem Theory Comput 2010, 6 (9), 2866-2871. [PubMed: 20844599]

6. van Gunsteren WF; Berendsen HJ; Hermans J; Hol WG; Postma JP, Computer simulation of the dynamics of hydrated protein crystals and its comparison with x-ray data. Proceedings of the National Academy of Sciences of the United States of America 1983, 80 (14), 4315-9. [PubMed: 6576339]

7. Pettitt BM; Makarov VA; Andrews BK, Protein hydration density: theory, simulations and crystallography. Curr Opin Struct Biol 1998, 8 (2), 218-21. [PubMed: 9631296]

8. Higo J; Nakasako M, Hydration structure of human lysozyme investigated by molecular dynamics simulation and cryogenic X-ray crystal structure analyses: On the correlation between crystal water sites, solvent density, and solvent dipole. Journal of computational chemistry 2002, 23 (14), 13231336. [PubMed: 12214315]

9. Altan I; Fusco D; Afonine PV; Charbonneau P, Learning about Biomolecular Solvation from Water in Protein Crystals. J Phys Chem B 2018, 122 (9), 2475-2486. [PubMed: 29397724]

10. Wall ME, Internal protein motions in molecular-dynamics simulations of Bragg and diffuse X-ray scattering. IUCrJ 2018, 5 (Pt 2), 172-181.

11. Berman H; Henrick K; Nakamura H, Announcing the worldwide Protein Data Bank. Nat Struct Mol Biol 2003, 10 (12), 980-980.

12. Nakamura A; Ishida T; Kusaka K; Yamada T; Fushinobu S; Tanaka I; Kaneko S; Ohta K; Tanaka H; Inaka K; Higuchi Y; Niimura N; Samejima M; Igarashi K, "Newton's cradle" proton relay with amide-imidic acid tautomerization in inverting cellulase visualized by neutron crystallography. Science advances 2015, 1 (7), e1500263. [PubMed: 26601228]

13. Blakeley MP; Kalb AJ; Helliwell JR; Myles DAA, The 15-K neutron structure of saccharide-free concanavalin A. Proceedings of the National Academy of Sciences of the United States of America 2004, 101 (47), 16405-16410. [PubMed: 15525703]

14. Chen JCH; Hanson BL; Fisher SZ; Langan P; Kovalevsky AY, Direct observation of hydrogen atom dynamics and interactions by ultrahigh resolution neutron protein crystallography. 
Proceedings of the National Academy of Sciences of the United States of America 2012, 109 (38), 15301-15306. [PubMed: 22949690]

15. Blakeley MP, Neutron macromolecular crystallography. Crystallography Reviews 2009, 15 (3), 157-218.

16. Ashkar R; Bilheux HZ; Bordallo H; Briber R; Callaway DJE; Cheng X; Chu X-Q; Curtis JE; Dadmun M; Fenimore P; Fushman D; Gabel F; Gupta K; Herberle F; Heinrich F; Hong L; Katsaras J; Kelman Z; Kharlampieva E; Kneller GR; Kovalevsky A; Krueger S; Langan P; Lieberman R; Liu Y; Losche M; Lyman E; Mao Y; Marino J; Mattos C; Meilleur F; Moody P; Nickels JD; O'Dell WB; O'Neill H; Perez-Salas U; Peters J; Petridis L; Sokolov AP; Stanley C; Wagner N; Weinrich M; Weiss K; Wymore T; Zhang Y; Smith JC, Neutron scattering in the biological sciences: progress and prospects. Acta Crystallographica Section D 2018, 74 (12), 1129-1168.

17. Adams PD; Afonine PV; Bunkoczi G; Chen VB; Davis IW; Echols N; Headd JJ; Hung LW; Kapral GJ; Grosse-Kunstleve RW; McCoy AJ; Moriarty NW; Oeffner R; Read RJ; Richardson DC; Richardson JS; Terwilliger TC; Zwart PH, PHENIX: a comprehensive Python-based system for macromolecular structure solution. Acta Crystallogr D Biol Crystallogr 2010, 66 (Pt 2), 213-21. [PubMed: 20124702]

18. Berendsen HJC; van der Spoel D; van Drunen R, GROMACS: A message-passing parallel modecular dynamics implementation. Comput Phys Comm 1995, 91, 43-56.

19. Lindorff-Larsen K; Piana S; Palmo K; Maragakis P; Klepeis JL; Dror RO; Shaw DE, Improved side-chain torsion potentials for the Amber ff99SB protein force field. Proteins 2010, 78 (8), 1950-8. [PubMed: 20408171]

20. Jorgensen WL; Chandrasekhar J; Madura JD; Impey RW; Klein ML, Comparison of Simple Potential Functions for Simulating Liquid Water. Journal of Chemical Physics 1983, 79 (2), 926935.

21. Wang JM; Wolf RM; Caldwell JW; Kollman PA; Case DA, Development and testing of a general amber force field. Journal of computational chemistry 2004, 25 (9), 1157-1174. [PubMed: 15116359]

22. Kirschner KN; Yongye AB; Tschampel SM; Gonzalez-Outeirino J; Daniels CR; Foley BL; Woods RJ, GLYCAM06: a generalizable biomolecular force field. Carbohydrates. Journal of computational chemistry 2008, 29 (4), 622-55. [PubMed: 17849372]

23. Wall ME; Van Benschoten AH; Sauter NK; Adams PD; Fraser JS; Terwilliger TC, Conformational dynamics of a crystalline protein from microsecond-scale molecular dynamics simulations and diffuse X-ray scattering. Proceedings of the National Academy of Sciences of the United States of America 2014, 111 (50), 17887-92. [PubMed: 25453071]

24. Lexa KW; Carlson HA, Full Protein Flexibility Is Essential for Proper Hot-Spot Mapping. Journal of the American Chemical Society 2011, 133 (2), 200-202. [PubMed: 21158470]

25. Grosse-Kunstleve RW; Sauter NK; Moriarty NW; Adams PD, The Computational Crystallography Toolbox: crystallographic algorithms in a reusable software framework. J Appl Crystallogr 2002, 35, 126-136.

26. Winn MD; Ballard CC; Cowtan KD; Dodson EJ; Emsley P; Evans PR; Keegan RM; Krissinel EB; Leslie AG; McCoy A; McNicholas SJ; Murshudov GN; Pannu NS; Potterton EA; Powell HR; Read RJ; Vagin A; Wilson KS, Overview of the CCP4 suite and current developments. Acta Crystallogr D Biol Crystallogr 2011, 67 (Pt 4), 235-42. [PubMed: 21460441]

27. Mattos C; Ringe D, Locating and characterizing binding sites on proteins. Nat Biotechnol 1996, 14 (5), 595-599. [PubMed: 9630949]

28. Xue Y; Skrynnikov NR, Ensemble MD simulations restrained via crystallographic data: accurate structure leads to accurate dynamics. Protein Sci 2014, 23 (4), 488-507. [PubMed: 24452989]

29. Wlodawer A; Minor W; Dauter Z; Jaskolski M, Protein crystallography for non-crystallographers, or how to get the best (but not more) from published macromolecular structures. FEBS J 2008, 275 (1), 1-21.

30. Janowski PA; Cerutti DS; Holton J; Case DA, Peptide crystal simulations reveal hidden dynamics. J Am Chem Soc 2013, 135 (21), 7938-48. [PubMed: 23631449] 
31. Janowski PA; Liu C; Deckman J; Case DA, Molecular dynamics simulation of triclinic lysozyme in a crystal lattice. Protein Sci 2016, 25 (1), 87-102. [PubMed: 26013419]

32. Meinhold L; Merzel F; Smith JC, Lattice dynamics of a protein crystal. Phys Rev Lett 2007, 99 (13), 138101. [PubMed: 17930640]

33. Meinhold L; Smith JC, Correlated dynamics determining X-ray diffuse scattering from a crystalline protein revealed by molecular dynamics simulation. Phys Rev Lett 2005, 95 (21), 218103. [PubMed: 16384188]

34. Meinhold L; Smith JC, Fluctuations and correlations in crystalline protein dynamics: a simulation analysis of Staphylococcal nuclease. Biophys J 2005, 88 (4), 2554-63. [PubMed: 15681654]

35. Meinhold L; Smith JC, Protein dynamics from X-ray crystallography: anisotropic, global motion in diffuse scattering patterns. Proteins 2007, 66 (4), 941-53. [PubMed: 17154425]

36. Keedy DA; van den Bedem H; Sivak DA; Petsko GA; Ringe D; Wilson MA; Fraser JS, Crystal cryocooling distorts conformational heterogeneity in a model Michaelis complex of DHFR. Structure 2014, 22 (6), 899-910. [PubMed: 24882744]

37. Thomaston JL; Woldeyes RA; Nakane T; Yamashita A; Tanaka T; Koiwai K; Brewster AS; Barad BA; Chen Y; Lemmin T; Uervirojnangkoorn M; Arima T; Kobayashi J; Masuda T; Suzuki M; Sugahara M; Sauter NK; Tanaka R; Nureki O; Tono K; Joti Y; Nango E; Iwata S; Yumoto F; Fraser JS; DeGrado WF, XFEL structures of the influenza M2 proton channel: Room temperature water networks and insights into proton conduction. Proceedings of the National Academy of Sciences of the United States of America 2017, 114 (51), 13357-13362. [PubMed: 28835537]

38. Alexov E; Mehler EL; Baker N; Baptista A; Huang Y; Milletti F; Erik Nielsen J; Farrell D; Carstensen T; Olsson MHM; Shen JK; Warwicker J; Williams S; Word JM, Progress in the prediction of pKa values in proteins. Proteins: Structure, Function, and Bioinformatics 2011, 79 (12), 3260-3275.

39. Emsley P; Lohkamp B; Scott WG; Cowtan K, Features and development of Coot. Acta Crystallogr D Biol Crystallogr 2010, 66 (Pt 4), 486-501. [PubMed: 20383002] 


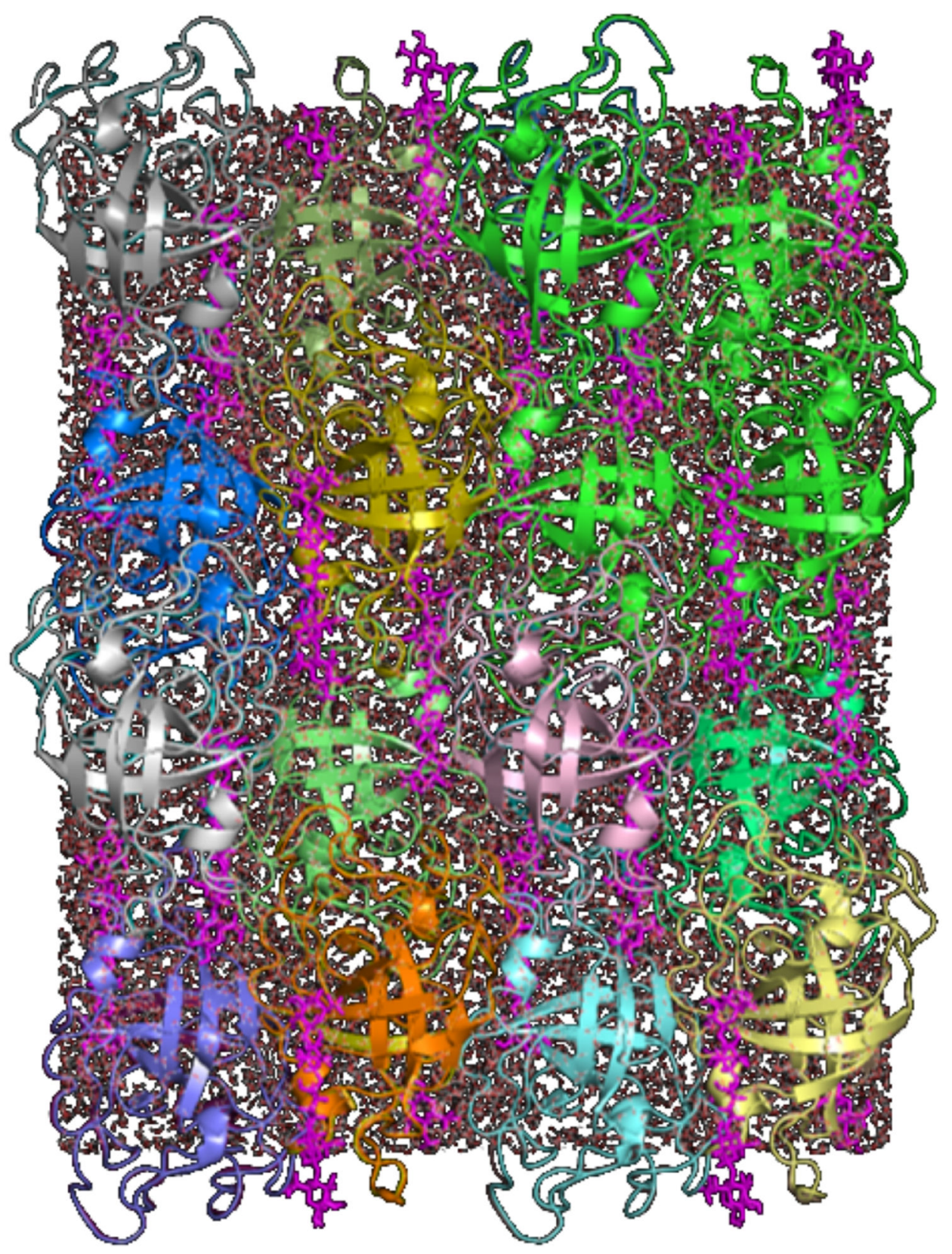

Figure 1.

Model of a $2 \times 2 \times 2$ periodic supercell of crystalline $\mathrm{EG}$, using the $\mathrm{NaCl}$ solvent model (Methods). The proteins are rendered using cartoon ribbons of various colors. The cellopentaose ligand is rendered using magenta sticks. Waters are rendered using sticks. 

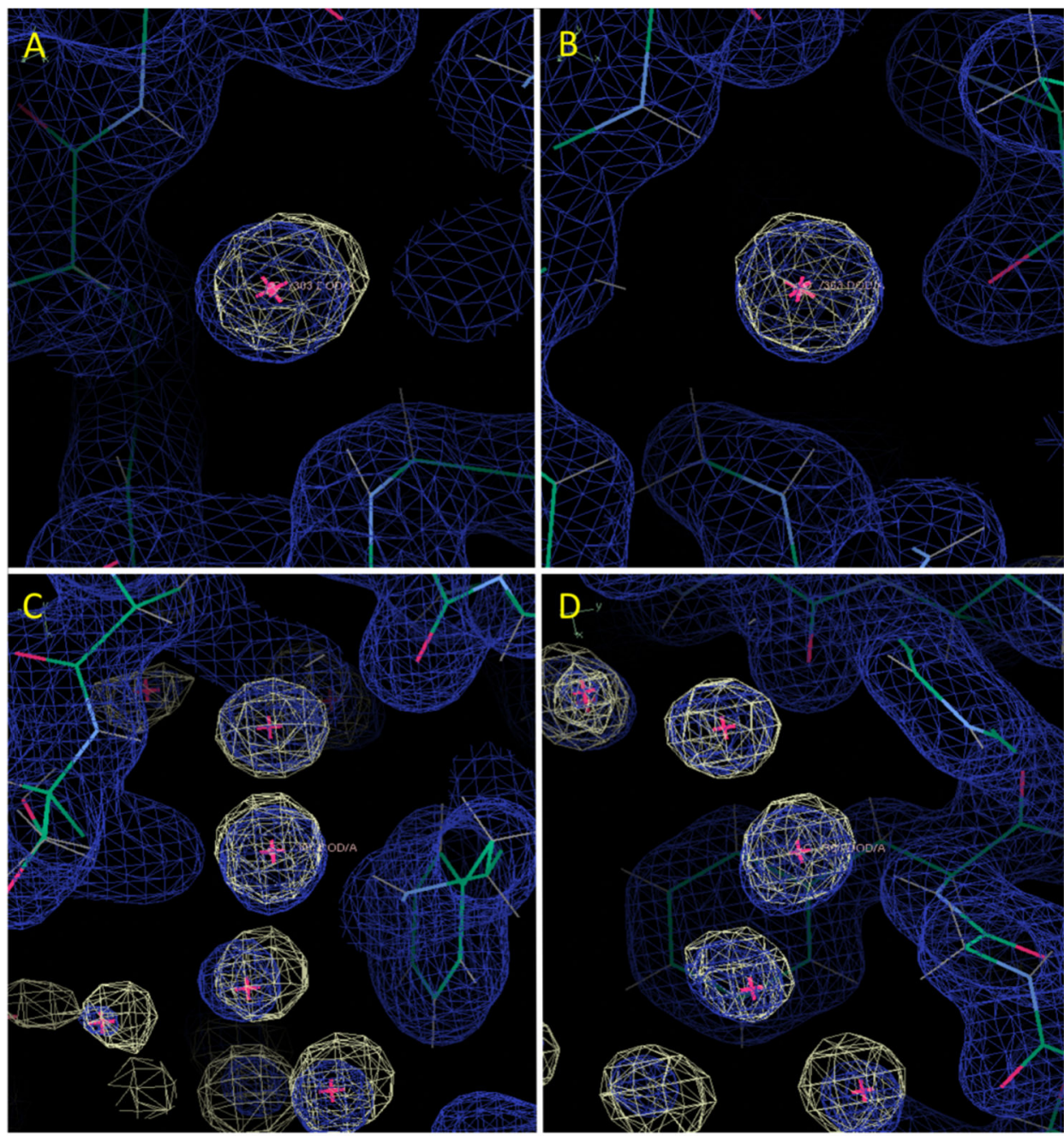

Figure 2.

Comparison of restrained $\mathrm{NaCl}$ model $\mathrm{MD}$ water electron density to crystallographic water positions and the X-ray 2Fo-Fc map. (A) View centered on buried water 303, which is isolated from other waters. (B) The same as (A), rotated roughly 90 degrees about the y-axis (vertical). (C) View centered on water 305, part of a connected water network. (D) The same as $(\mathrm{C})$, rotated roughly 90 degrees about the y-axis. The MD water density is rendered using a yellow wireframe at a level of 3 -sigma. The $2 \mathrm{Fo}-\mathrm{Fc}$ map is sigma-normalized and is shifted to have a mean of zero. It is rendered using a blue wireframe at a level of 1-sigma. The crystal water positions are indicated by magenta crosses. The panels were created using $\operatorname{Coot}^{39}$. 


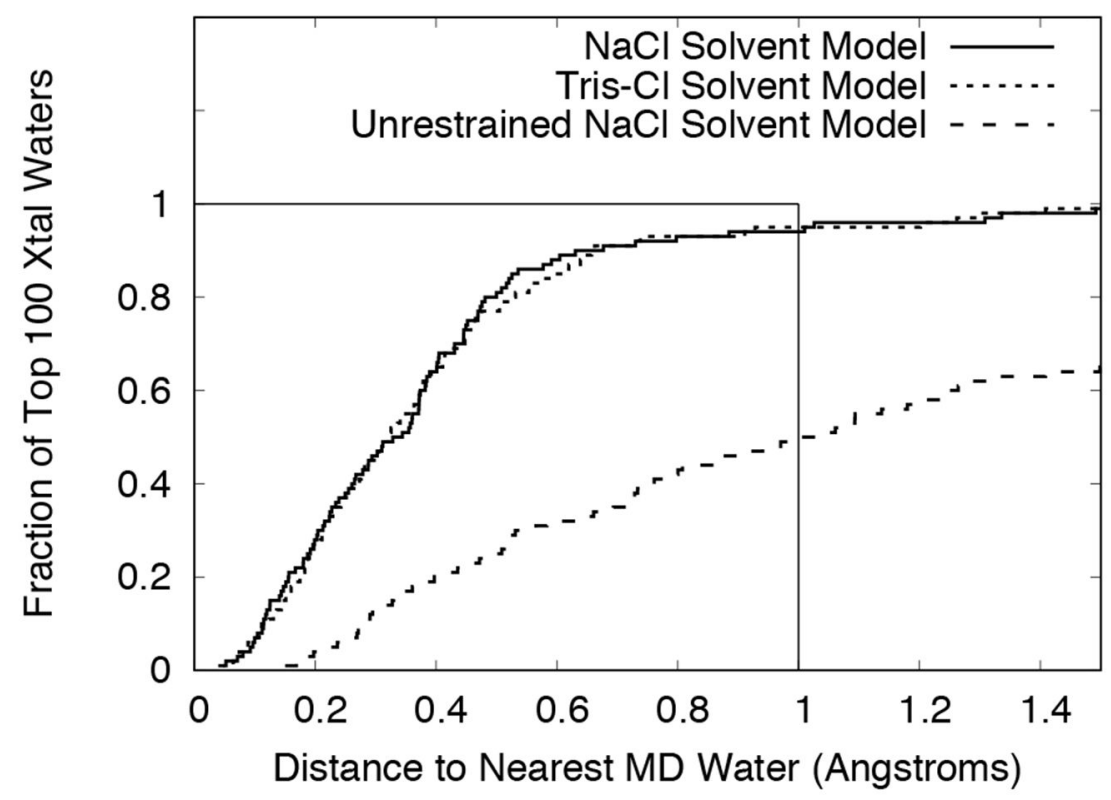

Figure 3.

Cumulative distribution of distances between crystallographic waters and peaks in the MD water electron density map. The results were computed using the top 100 crystallographic waters, with residue numbers 301-400. Results for the restrained $\mathrm{NaCl}$ model are indicated using a solid line, results for the restrained Tris- $\mathrm{Cl}$ model are indicated using a dotted line, and results for the unrestrained $\mathrm{NaCl}$ model are indicated using a dashed line. The boxed region bounded by $1 \AA$ on the $\mathrm{x}$-axis and a fraction of 1 on the $y$-axis is indicated using a thin solid line. 

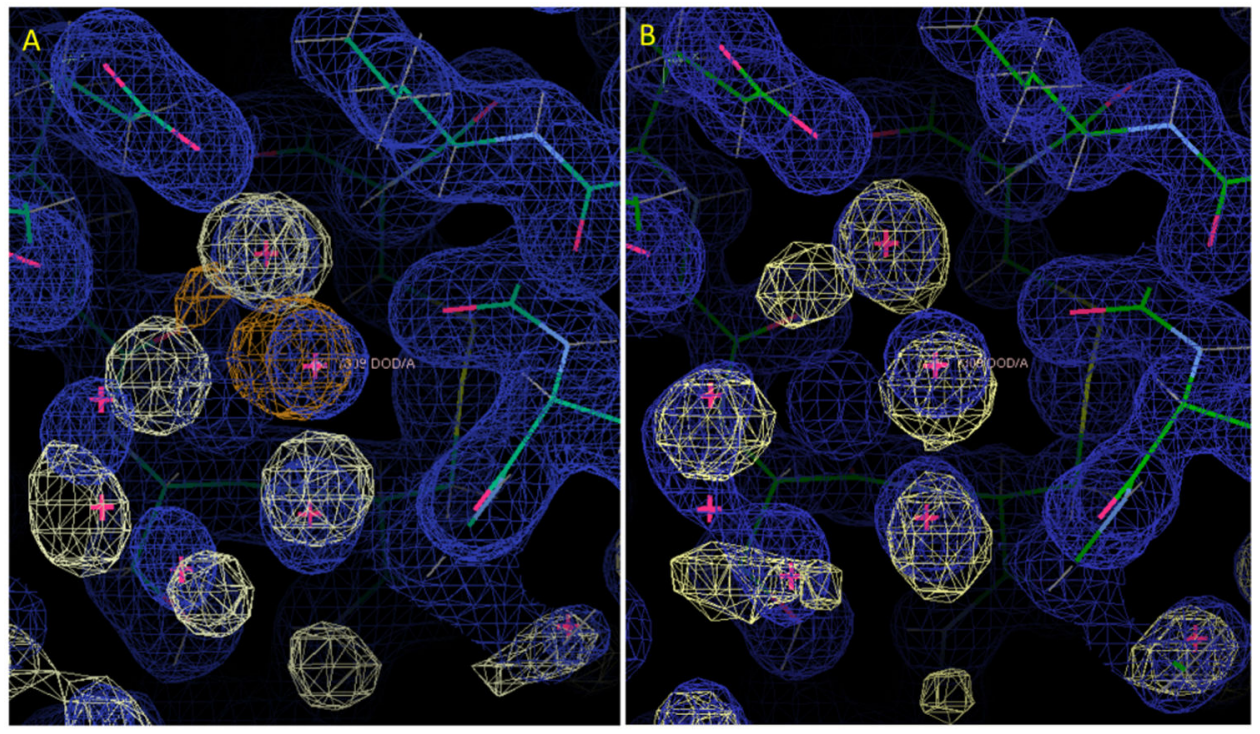

Figure 4.

Comparison of alternative solvent models in the neighborhood of water 309. (A) Restrained $\mathrm{NaCl}$ model. (B) Restrained Tris- $\mathrm{Cl}$ model in a similar orientation. Rendering of the MD water and X-ray 2Fo-Fc maps are as in Fig. 2. Panel (A) also includes the Na+ density, rendered in orange at a level of 8-sigma. In the $\mathrm{NaCl}$ model (A), the position of water 309 is occupied by a $\mathrm{Na}+$ ion, and there are substantial differences between the MD and crystallographic water structure nearby. In the Tris-Cl model (B), the MD water structure overlaps water 309, and the neighboring water structure is more similar between the MD and the crystallographic water structure. The panels were created using $\operatorname{Coot}^{39}$. 

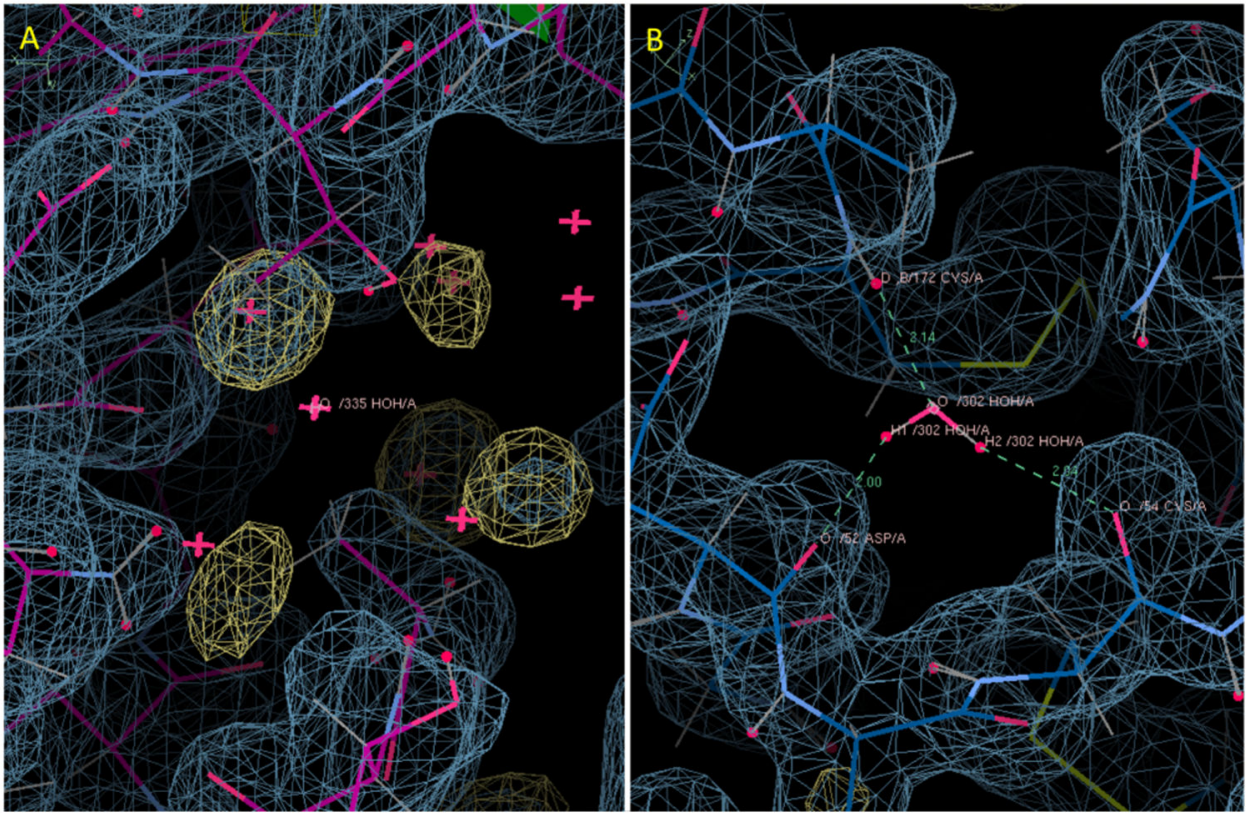

Figure 5.

Two types of water structure discrepancy for the unrestrained MD model. (A) Solventaccessible crystallographic water 335. MD water density is missing and some nearby waters deviate from the MD water density. (B) Buried water 302. MD water density is missing. Favorable H-bond interactions with the protein are indicated using dashed lines. In both panels, water MD density is shown using a yellow wireframe at a level of 2-sigma, and total MD density including the protein is shown using a cyan wireframe at a level of 1-sigma. The panels were created using $\mathrm{Coot}^{39}$. 


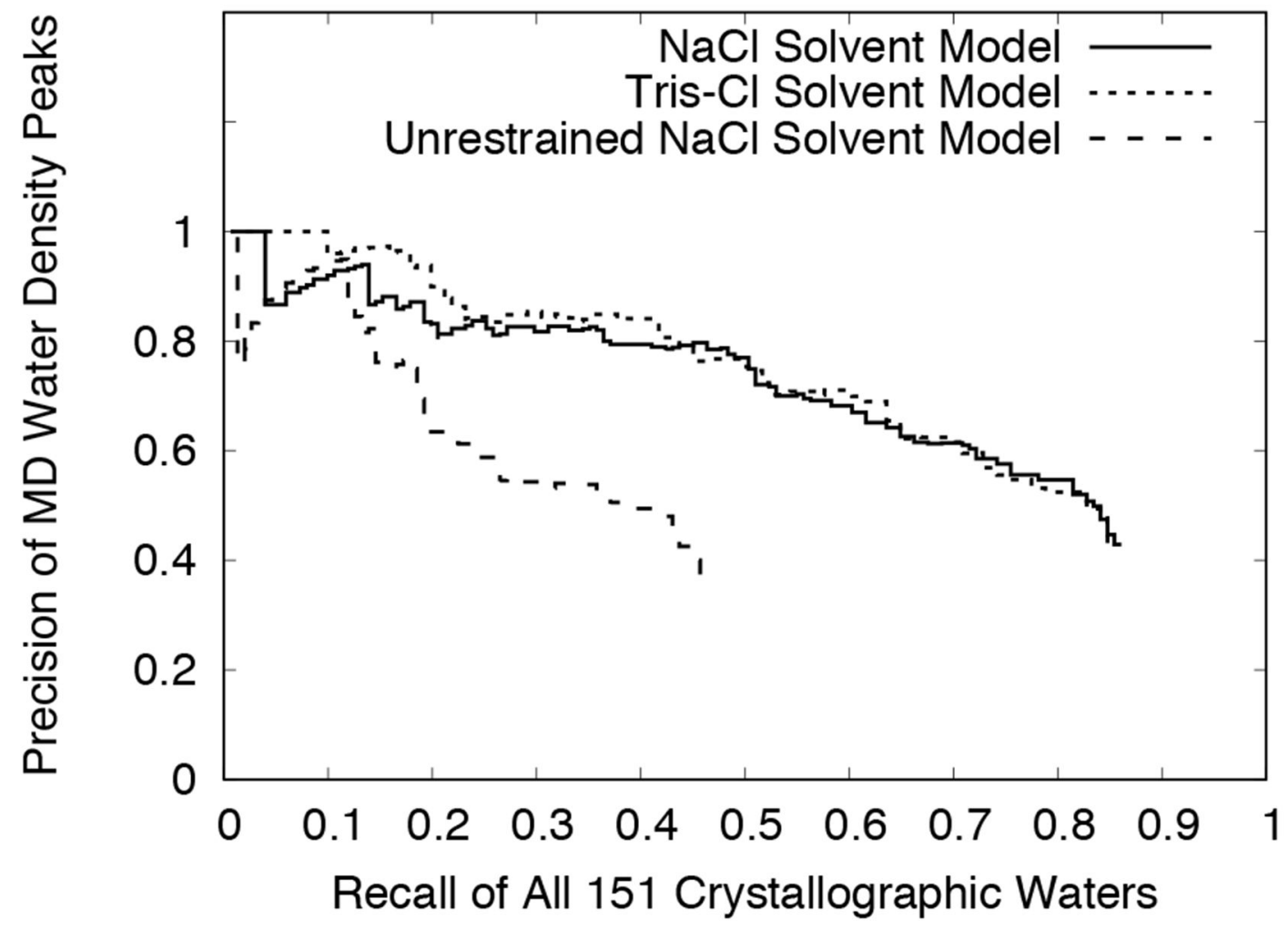

Figure 6.

Precision vs. recall using increasing MD water density peak thresholds. The peak threshold increases from right to left, as the recall decreases (Supporting Fig. S1). Statistics were computed using a cutoff distance of $1.0 \AA$. Results are shown for the last $10 \mathrm{~ns}$ of the $\mathrm{NaCl}$ and Tris- $\mathrm{Cl}$ solvent models, and for the first $100 \mathrm{~ns}$ of the unrestrained $\mathrm{NaCl}$ solvent model. 

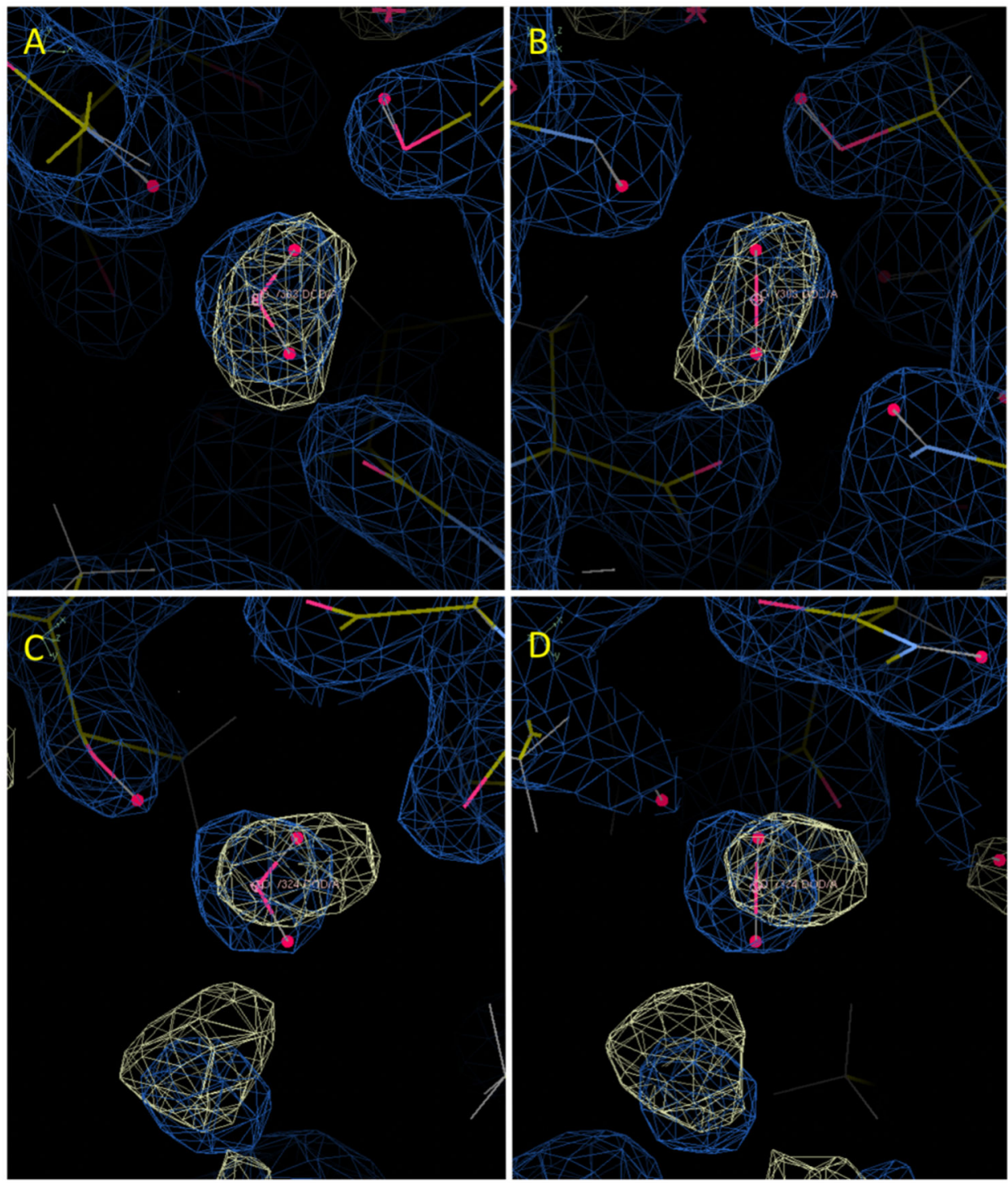

Figure 7.

Comparison of the restrained $\mathrm{NaCl}$ model $\mathrm{MD}$ water neutron scattering density to the crystallographic water orientation and the neutron $2 \mathrm{Fo}-\mathrm{Fc}$ map. (A) View centered on water 303. (B) the same as (A), rotated roughly 90 degrees about the y-axis. (C) View centered on water 328. (D) the same as (C), rotated roughly 90 degrees about the y-axis. The MD model and 2Fo-Fc maps are rendered as in Fig. 2. The panels were created using $\mathrm{Coot}^{39}$. 
Table I.

Recall of crystallographic waters for the MD water models. Units are percentages (\%).

\begin{tabular}{llll}
\hline & \multicolumn{3}{l}{ Recall of top $\mathbf{1 0 0}$ waters (all 151 waters) } \\
& $\mathbf{0 . 5} \AA^{\boldsymbol{e}}$ & $\mathbf{1 . 0} \AA^{f}$ & $\mathbf{1 . 4} \AA^{\boldsymbol{g}}$ \\
$\mathrm{NaCl}(\mathrm{R})^{a}$ & $80(66)$ & $94(86)$ & $98(93)$ \\
$\mathrm{Tris}-\mathrm{Cl}(\mathrm{R}){ }^{b}$ & $77(64)$ & $95(85)$ & $98(93)$ \\
$\mathrm{NaCl}(\mathrm{U}, \mathrm{F}){ }^{C}$ & $25(21)$ & $50(46)$ & $62(56)$ \\
$\mathrm{NaCl}(\mathrm{U}, \mathrm{L})^{d}$ & $18(15)$ & $42(36)$ & $51(42)$ \\
\hline
\end{tabular}

a Last $10 \mathrm{~ns}$ section of the restrained $\mathrm{NaCl}$ simulation.

$b_{\text {Last }} 10 \mathrm{~ns}$ section of the restrained Tris-Cl simulation.

${ }^{c}$ First $100 \mathrm{~ns}$ section of the unrestrained $\mathrm{NaCl}$ solvent $\mathrm{MD}$ simulation.

$d_{\text {Last }} 100 \mathrm{~ns}$ section of the unrestrained $\mathrm{NaCl}$ solvent MD simulation.

e Percentage of waters that have an MD peak within $0.5 \AA$.

$f_{\text {Percentage of waters that have an MD peak within } 1.0 \AA .}$

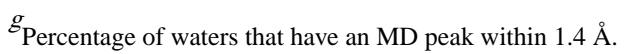

